\title{
A VLSI MODEL OF THE BAT LATERAL SUPERIOR OLIVE FOR AZIMUTHAL ECHOLOCATION
}

\author{
R. Z. Shi $i^{1,2}$ and T. K. Horiuchi $i^{1,2,3}$ \\ ${ }^{1}$ Electrical and Computer Engineering Department \\ ${ }^{2}$ Institute for Systems Research \\ ${ }^{3}$ Neuroscience and Cognitive Science Program \\ University of Maryland, College Park, MD 20742,USA
}

\begin{abstract}
Bats have long been the envy of engineers due to their ability to use echolocation to fly with speed and agility through complex 3D environments. By understanding the neurobiological basis for echolocation, we hope to emulate the efficient implementation demonstrated by nature. Bats use interaural level differences (ILD) as their primary cue for azimuthal echolocation. The Lateral Superior Olive (LSO) is bat's first ILD processing center and plays an important role. We have designed a CMOS VLSI circuit based neuromorphic system that mimics ILD processing in the bat LSO. In this paper,we propose a simple spiking neural model of LSO cells, and a VLSI implementation of an array of cells representing the LSO population.
\end{abstract}

\section{INTRODUCTION}

Bats localize objects by emitting ultrasonic pulses and processing the resulting echoes from objects. This mechanism is called echolocation. Their small head size and the use of high frequency sound makes the interaural level differences (ILDs) their primary cue for azimuthal echolocation. In bats, ILDs are known to be coded at the earliest stage of binaural processing by neurons in the lateral superior olive (LSO). The LSO receives its principal excitatory (E) inputs from the ipsilateral ear and inhibitory (I) inputs from the contralateral ear. Therefore, neurons in LSO are mainly of the IE type. Fig. 1 shows one cell's sensitivity to ILD (or IID, interaural intensity difference) and corresponding raster plots for a typical LSO ILD-sensitive neuron. The asterisk in Fig. 1 is defined as the ILD of complete inhibition. The ILD of complete inhibition defines the LSO cell's sensitivity to ILD which varies among different LSO cells and thus codes azimuthal information.

There are only a few ILD models that consider detailed biological structures. Zacksenhouse et al. proposed a computational model of single LSO units [1] that applies point process theory for modeling responses in the LSO of cats to transient and sustained input. The compartmental model is not that easily extended to multi-layer networks. Reed and Blum proposed a specific neural network model for the computation and encoding of the azimuthal information by the LSO [2]. However, their model used steady states firing rate and did not consider timing issue between excitation and inhibition. Recently, Horiuchi and Hynna initiated a spike-based VLSI modeling study of the bat ILD system [3] and this paper continues their work by emphasizing more detailed modeling of the LSO and an array of circuits with different parameters.

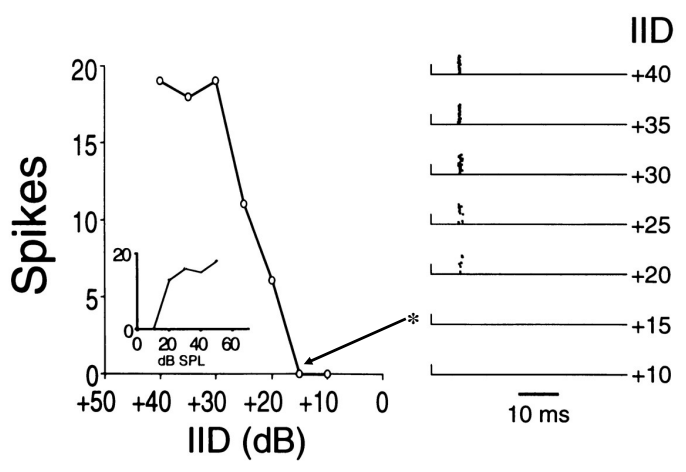

Fig. 1. Interaural level difference (IID in the figure) functions and corresponding raster plots for a typical ILDsensitive neuron from the LSO. Positive ILDs indicate a greater intensity at the excitatory ear. Stimuli were 2-ms long, 10-kHz downward frequency sweeps centered at each unit's characteristic frequency. Intensity to the excitatory ear was fixed at $20 \mathrm{~dB}$ above threshold, whereas the intensity to the inhibitory ear was varied. Each ILD was presented 20 times in pseudo random order. Inset: rate-level functions for each cell. The ILD at asterisk of each panel is defined as ILD of complete inhibition. Revised from [4]. 


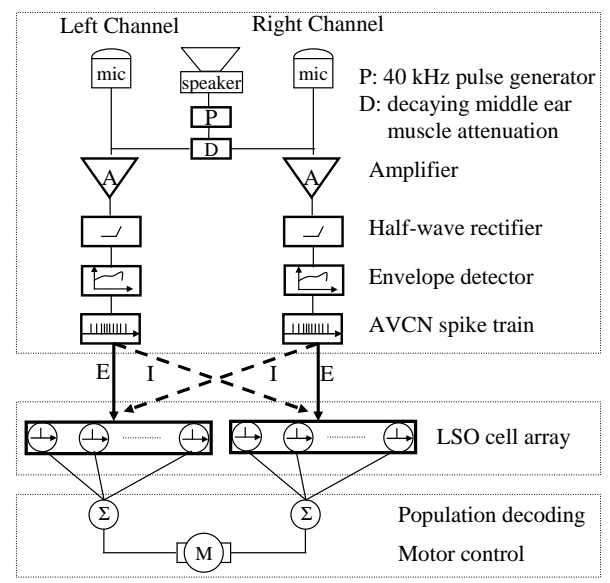

Fig. 2. Block diagram of the VLSI based hardware system. Top box: front end by board design. Middle box: LSO population by chip design. Bottom box: post-processing by board design.

\section{SYSTEM, MODEL, AND CIRCUIT}

\subsection{System Architecture}

Our neuromorphic azimuthal echolocation system consists of three parts: a sensory front-end that provides inputs to the LSO cell, a muticell LSO chip that generates population responses corresponding to different ILD inputs, and a back-end circuit that post-processes the LSO population response to drive a tracking motor system. Fig. 2 shows the block diagram of the system.

The sonar front end transmits short $40 \mathrm{kHz}$ pulses, receives, and processes the echo signals. The received echo signals are amplified, half-wave rectified and the envelopes are then extracted. The last stage of the front end is a spike generator that represents the spike train from populations of anteroventral cochlear nucleus (AVCN). This front end is similar to that reported in [3].

The back-end extracts azimuth information from the LSO spike population response. The decoded azimuth information is then transformed into signals that control the motor tracking system. We omit details of the front and back end components of the system in this paper. In the following, we describe the LSO model and chip implementation.

\subsection{LSO model}

While exact information about excitatory and inhibitory convergence onto the bat LSO data is not available, experiments in the gerbil suggest that there are about ten excitatory and eight inhibitory synapses on a single LSO cell [5], [1]. We model each LSO IE cell as a spiking neuron with one excitatory synapse and one inhibitory synapse for simplicity. Zacksenhouse and Johnson's modeling work has one sig- nificant result: inhibition in LSO is basically a shunting effect [1]. As our VLSI synapse model uses synaptic current rather than conductance, the clamping effect of inhibition is naturally implemented by our inhibitory synapse which shunts the membrane capacitance to the reversal potential of zero volts. Above zero, the synapse acts like a hyperpolarizing inhibition.

We may apply the Gerstner's spike response model [6] to formalize our ILD algorithm as follows. The state of an LSO cell i can be described by its membrane voltage, denoted by $u_{i}$. The set of all firing times of LSO cell $\mathrm{i}$ is denoted by

$$
F_{i}=\left\{t_{i}^{(f)} ; 1 \leq f \leq n\right\}=\left\{t \mid u_{i}(t)=\theta\right\}
$$

while $\theta$ is the firing threshold of the LSO cell. For a specific ILD, assume the spike train for the excitatory input to the LSO is

$$
E=\left\{t^{(f)} ; 1 \leq f \leq N_{e}\right\}
$$

and inhibitory spike train

$$
I=\left\{t^{(f)} ; 1 \leq f \leq N_{i}\right\}
$$

The state $u_{i}(t)$ of LSO neuron $\mathrm{i}$ at time $\mathrm{t}$ is then given by:

$$
\begin{aligned}
u_{i}(t)= & {\left[\sum_{t_{i}^{(f)} \in F_{i}} \eta_{i}\left(t-t_{i}^{(f)}\right)+\sum_{t^{(f)} \in E} \operatorname{exc}\left(t-t^{(f)}\right)\right.} \\
& \left.-\sum_{t^{(f)} \in I} i n h\left(t-t^{(f)}\right)\right]^{+}
\end{aligned}
$$

The kernel $\eta_{i}$ is the mathematical description of refractory function of the LSO cell i. The kernels $\operatorname{exc}\left(t-t^{(f)}\right)$ and $i n h\left(t-t^{(f)}\right)$ are the contribution of the excitatory and inhibitory synapses, respectively. The time to the first LSO spike, as determined by Eqs. (1)-(4), defines each LSO cell's ILD sensitivity.

In short, our model simplifies ILD computation at LSO as a linear superposition of excitation and inhibition and a nonlinear shunting effect from inhibition when the membrane voltage is zero. Such a spiking neural model emphasizes the importance of timing between excitatory and inhibitory spike train, and the output of the LSO cell carries this timing information to the next stage. Park et al.'s experimental work on bat LSO concludes that it is the difference in latencies, in addition to thresholds, that determines the ILD of complete inhibition [7]. This is exactly what our model does.

\subsection{The LSO chip}

The LSO circuit schematic is shown in Fig. 3. The threshold $\theta$ is determined by Vlim and Vdd. Vo is the LSO spike output. The refractory kernel $\eta_{i}\left(t-t_{i}^{(f)}\right)$ is controlled by 


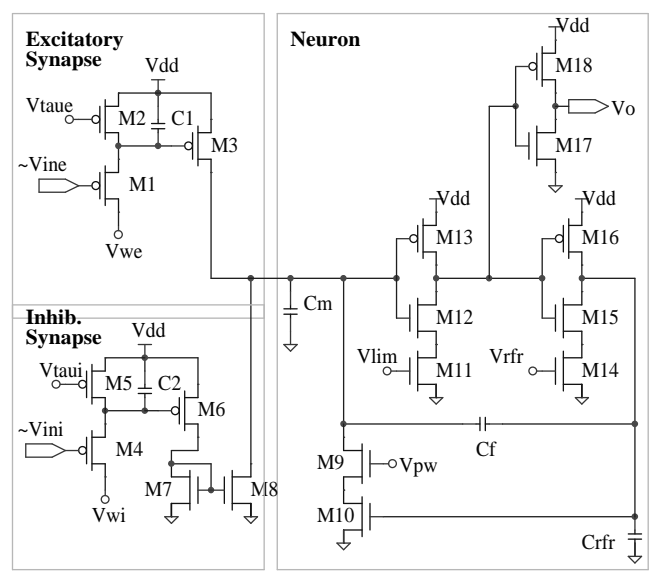

Fig. 3. Schematics of LSO circuit model.

the dynamics of Crfr, together with Vpw and transistor M9, M10. Cf is to set the positive feedback once the neuron is firing, as explained in [8].

We used a synapse first introduced by Lazzaro and Wawrzynek [9]. This synapse circuit has the advantage of small size and ease of control. When $\mathrm{Vw}$ is set below the transistor threshold, this synapse produces a clean exponential decay in synaptic current, which is an important virtue of the biological counterpart. The downside of this synapse circuit is that it does not implement linear temporal summation of rapid bursts of input spikes. We can, however, design the synapse to have a small time constant such that the synaptic current decays to zero before a new presynaptic spike arrives. This ensures a linear summation of inputs at the membrane capacitor. Vwe and Vwi set the weight of the excitatory and inhibitory synapse, respectively. Vtaue and Vtaui set the time constant of synapse output current.

Two chips are used in the echolocation system, the left LSO chip and the right LSO chip. Each chip contains 16 IE cells. All the IE cells receive the same excitatory input (E) and the same inhibitory input (I), have the same inhibitory synapse weight (Vwi), and the same synapse time constants (Vtaue and Vtaui). The different threshold responses among the 16 IE cells are achieved by assigning different excitatory synapse weights, determined by different tap points on a polysilicon resistor line. The circuit was fabricated in a commercially-available $1.5 \mu \mathrm{m}$ technology.

\section{TESTING RESULTS}

We report chip testing results as shown in Fig. 4-6. Figs. 4 is the measured ILD function (ILD tuning curve) for 15 cells in the LSO chip. The input signals to the front end are two $40 \mathrm{kHz}$ AM signals with durations of $1 \mathrm{msec}$, where the magnitude varies between the two sides. 15 LSO cells are tested under 10 trials per ILD input. Excitatory synaptic

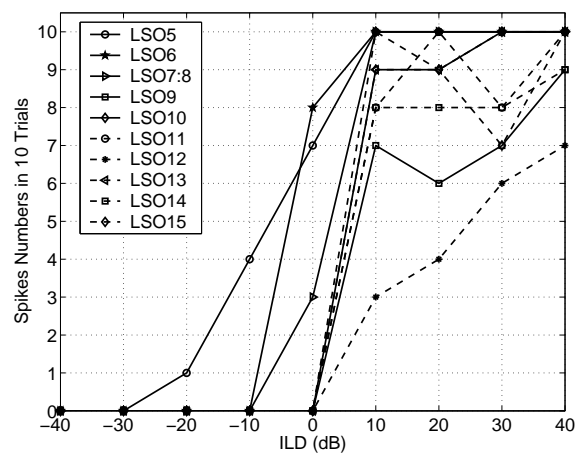

Fig. 4. Measured ILD function. LSO cells are tested under 10 trials per ILD input.

strength decreases as the LSO cell number increases. LSO cell 1-4 (not shown) fire one spike for all the ILD range.

Fig. 5 shows a particular cell's response in the left LSO chip to a vertical cylinder, located about 10 degrees left and $70 \mathrm{~cm}$ away from the speaker. The top two panels show the spike train from the output of the front end. The third panel is the membrane voltage response of this LSO cell which fires at the threshold voltage of about 3 volts. The bottom panel shows the LSO spike output. Note that there is about $4 \mathrm{msec}$ of delay from the onset of speaker emission to the time zero in Fig. 5. For this LSO cell, the echo from the left (ipsilateral side) side is excitatory, and the echo from the right side (contralateral side) is inhibitory. We see clearly from Fig. 5 how excitation is summated and how inhibition is subtracted on the membrane voltage. Immediately after the LSO cell fires a spike, inputs to the cell have no effect due to the refractory period of the LSO circuit.

Fig. 6 shows the population response to the same cylinder target in three different directions with a range of 60 $\mathrm{cm}$. We see how the azimuthal location of a target is coded by the number of LSO cells that respond, and the relative timing of their spikes. This population response and timing information is conveyed to the ascending ILD pathway of bats for more complex processing. In our neuromorphic echolocation system illustrated in Fig. 2, this information has been used to extract azimuth to drive a tracking motor.

\section{SUMMARY}

We have presented a neuromorphic azimuthal echolocation system that mimics the ILD processing in the bat LSO. We have proposed a spiking neural model that is based on Park et al.'s bat LSO experimental results. Our model and circuit implementation are simple, but they capture the most important computation performed in the LSO. We are designing a linear summating synapse with initial results reported in [10]. We are also designing a multi-chip system that incorporates knowledge of the LSO, the dorsal nucleus of the lat- 

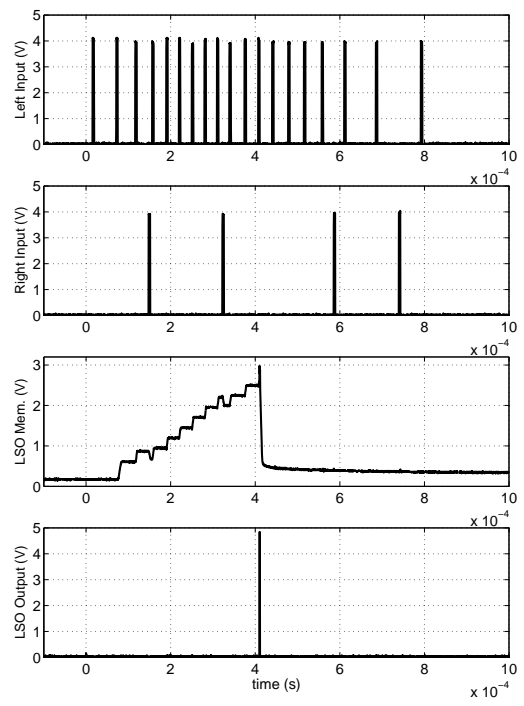

Fig. 5. Sample traces of a cell in left LSO chip. The object is located about 10 degrees left of center and $70 \mathrm{~cm}$ away. The top two panels shows the spike train from the output of the front end. The third panel is the membrane voltage response of this LSO cell, it fires at the threshold voltage about 3 volts. The bottom panel shows the LSO spike output.
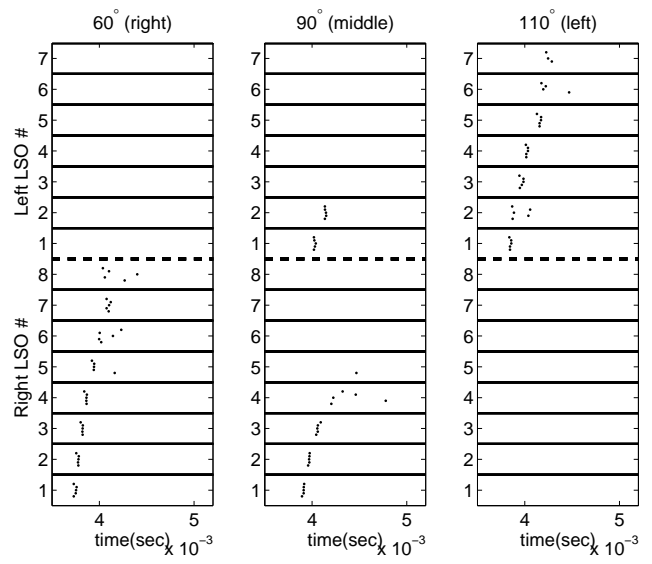

Fig. 6. Population response at 3 different locations. Spike rasters from seven cells in the left LSO chip and eight cells in the right LSO chip are shown with five trials at each location. In both chips, excitatory synaptic strength decreases with increasing LSO cell index. Time shown is relative to the onset of the pulse. Left panel: target is at 60 degrees (or 30 degs right of center). Middle panel: target is centered. Right panel: target is 110 degrees (or 20 degs left of center). eral lemniscus (DNLL), and the inferior colliculus (IC) for more complex ILD computation and exploring its impact on bat echolocation. ILD processing is not a computation unique to bats, it is a significant feature for localization in cats, gerbils, owls, monkeys and in humans. Our circuits, by extension, capture many of aspects of ILD computation in these systems as well.

\section{ACKNOWLEDGMENTS}

This work was supported by funding from DARPA (N0001400C0315) and the Air Force Office of Strategic Research (AFOSR F496200110415). We greatly thank Kaushik Ghose for his work on the PCB design of the back end circuit. We thank MOSIS for fabrication services in support of our neuromorphic analog VLSI course and teaching laboratory.

\section{REFERENCES}

[1] M. Zacksenhouse, D. H. Johnson, J. Williams, and C. Tsuchitani, "Single-neuron modeling of LSO unit responses," $J$. Neurophysiol., vol. 79, pp. 3098-3110, 1998.

[2] M. C. Reed and J. J. Blum, "A model for the computation and encoding of azimuthal information by the lateral superior olive," J. Acoust. Soc. Am., vol. 88, pp. 1442-1453, 1990.

[3] T. Horiuchi and K. Hynna, "Spike-based VLSI modeling of the ILD system in the echolocating bat," Neural Networks, vol. 14, pp. 755-762, 2001.

[4] T. J. Park, "IID sensitivity differs between two principle centers in the interaural intensity difference pathway: the LSO and the IC," J. Neurophysiol., vol. 79, pp. 2416-2431, 1998.

[5] D. H. Sanes, "An in vitro analysis of sound localization mechanisms in the gerbil lateral superior olive," J. Neurosci., vol. 10, pp. 3494-3506, 1990.

[6] W. Gerstner and W. M. Kistler, Spiking Neuron Models: Single Neurons, Populations, Plasticity, Cambridge Unvisity Press, Cambridge, UK, 2002.

[7] T. J. Park, P. Monsivais, and G. D. Pollak, "Processing of interaural intensity differences in the LSO: role of interaural threshold differences," J. Neurophysiol., vol. 77, pp. 2863$2878,1997$.

[8] C. Mead, Analog VLSI and Neural Systems, AddisonWesley, Reading, MA, 1989.

[9] J. P. Lazzaro, "Low-power silicon axons, neuons, and synapses," in Silicon Implementations of Pulse Coded Neural Networks, M. E. Zaghloul, J. L. Meador, and R. W. Newcomb, Eds., pp. 153-164. Kluwer Academic Publishers, Norwell, MA, 1994.

[10] R. Z. Shi and T. Horiuchi, "A summating, exponentiallydecaying CMOS synapse for spiking neural systems," in Advances in Neural Information Processing Systems. MIT Press, Cambridge, MA, (accepted) 2004. 